\title{
EL TIEMPO DE TRABAJO DE LAS CUIDADORAS INMIGRANTES DE PERSONAS MAYORES
}

\section{The working time of migrant caregivers for elderly people}

\author{
EVA MARTíN COPPOLA* \\ JESÚS ROGERO GARCÍA**
}

\section{Resumen}

Este artículo realiza una aproximación a las condiciones laborales de las trabajadoras inmigrantes que cuidan personas mayores en su domicilio, a través de su dimensión temporal: tiempo de trabajo y horarios. Se aplicó una encuesta piloto sobre uso del tiempo $(\mathrm{N}=24)$ y se realizaron 60 entrevistas en profundidad a cuidadoras inmigrantes en la Comunidad de Madrid (España) en 2008. Los resultados reflejan que la jornada laboral de las cuidadoras se sitúa muy por encima de la jornada ordinaria, con 75 horas semanales, que alcanzan las 131 horas en el caso de las internas. Se distinguen dos tipos de cuidadoras en función de las actividades que realizan: la «acompañanteama de casa», que desarrolla fundamentalmente actividades domésticas y de acompañamiento, y la «enfermera-ama de casa», cuyo núcleo diferenciador son los cuidados sociosanitarios y las ayudas personales en el hogar. Asimismo, se constata que el uso del tiempo semanal de las cuidadoras varía sustancialmente según régimen de trabajo. El trabajo concluye con una reflexión crítica sobre las condiciones laborales de este colectivo, que se pone en contraste con la regulación laboral española.

Palabras clave: cuidados domiciliarios, inmigración, uso del tiempo, dependencia.

\footnotetext{
* Eva Martín Coppola

Centro de Ciencias Humanas y Sociales

Consejo Superior de Investigaciones Científicas (CSIC)

C/ Albasanz 26-28. Despacho 3F6. 28037 Madrid

Teléfono: 916022781 Fax: 916022971

* Jesús Rogero García

Centro de Ciencias Humanas y Sociales

Consejo Superior de Investigaciones Científicas (CSIC)

C/ Albasanz 26-28. Despacho 3E10. 28037 Madrid

Teléfono: 916022753 Fax: 916022971

jesus.rogero@cchs.csic.es, jrogero@gmail.com
} 


\begin{abstract}
This article addresses the working conditions of migrants who care for elderly people in their own homes through a temporal dimension: working time and working schedule. We applied a pilot survey on time use $(\mathrm{N}=24)$ and conducted interviews with 60 immigrant caregivers in Madrid (Spain) in 2008. The main results indicate that the working hours of the caregivers are well above the average working day, with around 75 hours a week and even reaching the 131 hours in the case of the live-in caregivers. There are two types of caregivers in terms of their working activities: those who only carry out domestic activities and accompanying duties, and those who also provide nursing care and personal help at home. The weekly time use varies substantially depending on carers' employment status. The paper concludes with a critical reflection on the working conditions of this group and contrasts them with the Spanish labour regulations.
\end{abstract}

Keywords: Home care services, immigration, time use, dependency.

\title{
1. Introducción
}

La rápida expansión del cuidado a mayores por inmigrantes y sus múltiples derivaciones han generado recientemente un buen número de investigaciones en España, que reflejan la complejidad de la confluencia de problemáticas propias de la migración y de la dependencia. A pesar de estos avances, hay algunos elementos que dificultan el conocimiento sobre la situación de vida y trabajo de quienes provienen de otros países y se dedican a cuidar personas mayores. En concreto, este fenómeno está sujeto a una doble opacidad: por una parte, la derivada de una relación laboral informal que no pasa por el mercado y por otra, la que resulta de la privacidad del ámbito familiar.

Aunque no son abundantes, en los últimos años se han desarrollado en España algunas fuentes de datos que contienen información sobre cuidadores de origen extranjero. El módulo de empleados de hogar de la Encuesta de Apoyo a Mayores en España (IMSERSO, 2005b) es la fuente de representación nacional más reciente realizada en nuestro país con datos sobre cuidadoras remuneradas en el hogar. Tiene una muestra de 72 empleadas de hogar de nacionalidad no española y recoge información sobre frecuencia semanal, tiempo dedicado al cuidado, momento del día en que se desarrolla y actividades realizadas. Por su parte, la reciente aparición de la Encuesta Nacional de Inmigrantes 2008, aunque contiene abundante información sobre condiciones de vida y trabajo de este colectivo, no incluye entre sus categorías de ocupación el trabajo de cuidado a personas dependientes.

A pesar de la escasez de información estadística, en los últimos años se han realizado estudios monográficos de gran interés sobre el colectivo de trabajadoras inmigrantes en el sector de cuidados (Pérez Orozco et al., 2008; 
IMSERSO, 2005a, Martínez Buján, 2005 y 2006; Pla Julián, 2004; y Colectivo IOÉ, 2003, entre otros). Estos esfuerzos constituyen un indudable avance en la comprensión de este fenómeno. Entre otras cuestiones, estos estudios han aportado información sobre las actividades que realizan los inmigrantes en la atención a personas mayores. El cuidado implica un conjunto de tareas muy heterogéneo, que comprende la ayuda en actividades básicas de la vida diaria (ir al baño, comer, asearse, desplazarse en el hogar, etc.), actividades instrumentales de la vida diaria (limpiar la casa, cocinar, etc.) y actividades avanzadas de la vida diaria (participar en actividades de ocio, etc.). Sin embargo, hay cuestiones sobre el trabajo y la vida cotidiana de los cuidadores inmigrantes que merecen especial atención y que, hasta la fecha, no han sido abordadas por ninguna investigación de manera específica. Por ejemplo, ningún estudio aporta datos precisos sobre el uso del tiempo de las cuidadoras durante un ciclo semanal completo, y tampoco se ha analizado con precisión el tiempo que se dedica diariamente a cada una de las diferentes tareas que componen el cuidado.

Este artículo pretende contribuir al conocimiento de la realidad de estas trabajadoras a través de una nueva mirada, la proporcionada la información sobre uso del tiempo. Su objetivo es realizar un conocimiento exploratorio de las condiciones de vida y trabajo de los inmigrantes que cuidan a personas mayores en la Comunidad de Madrid, prestando especial atención a su dimensión temporal: tiempo de trabajo remunerado y relación trabajo remunerado-vida privada.

\section{Metodología}

Este trabajo analiza de manera complementaria información procedente de la Encuesta Piloto sobre Uso del Tiempo a Cuidadoras de Origen Extranjero en la Comunidad de Madrid 2008 (EPUTC, 2008) -un instrumento metodológico construido ad hoc para conocer el uso del tiempo de los cuidadores inmigrantes-y de 60 entrevistas en profundidad. La perspectiva cuantitativa tiene como antecedentes estudios previos sobre el uso del tiempo de cuidadores informales (Bittman et al., 2004; Pedrero, 2008) y permite visibilizar el tiempo dedicado por las cuidadoras a diferentes actividades, tanto durante su tiempo de trabajo como fuera del mismo. El objetivo del análisis cualitativo es conocer cómo estas trabajadoras experimentan su tiempo cotidiano, le atribuyen significado y diseñan estrategias para su organización.

El diseño muestral de ambas fuentes tiene representatividad cualitativa o intencional, es decir, trata de incluir la diversidad de situaciones o prácticas del objeto de estudio que han sido puestas de manifiesto por anteriores 
investigaciones. Se seleccionaron cuidadoras de origen extranjero en la Comunidad de Madrid que trabajaban en hogares privados y que cuidaban personas mayores de 64 años. Dado que no existe un censo ni directorio de esta población que permita seguir un muestreo convencional, el procedimiento de muestreo fue de bola de nieve a través de asociaciones de inmigrantes, ONGs y parroquias. La muestra se diseñó en función de la nacionalidad, el régimen de cuidado y el estado civil de las cuidadoras, tomando como principal referencia la Encuesta a Empleados de Hogar de Apoyo a Mayores (IMSERSO 2005b).

En el caso de la encuesta, se seleccionaron trabajadoras que cuidaban sólo a una persona mayor. Este criterio era imprescindible para establecer relaciones consistentes entre las características de la cuidadora y de la persona cuidada. La muestra final de la encuesta fue de 24 mujeres. El cuestionario incluyó dos instrumentos de recogida de información para captar diferentes aspectos del uso del tiempo: la encuesta de actividades, en la que las entrevistadas indicaron la frecuencia de realización y el tiempo dedicado a una serie de actividades durante el último día de trabajo (Tabla 1), y el diario de empleo del tiempo, que recogió secuencialmente información sobre las actividades desarrolladas durante una semana completa en intervalos de 15 minutos. El diario es la metodología de investigación sobre uso del tiempo con mayor aceptación internacional entre los expertos (Eurostat, 2008; ONU, 2006; INE, 2003). La elección de uno u otro instrumento para estimar el tiempo de trabajo de cuidado depende del criterio utilizado para definir trabajo. La estimación realizada a través del diario considera trabajo todo el tiempo que, por acuerdo con la persona dependiente o la familia, las cuidadoras deben permanecer en el hogar, incluidos los tiempos de presencia ${ }^{1}$. Parte del trabajo de cuidado consiste en estar disponible para proveer el servicio en el momento en que sea necesario. Profesionales como bomberos, enfermeras o vigilantes de seguridad tienen tiempos de trabajo similares a los de las cuidadoras: deben estar preparados para una posible intervención, cuya planificación no es posible por su carácter imprevisible. Por su parte, la estimación a través de la encuesta de actividades contabiliza únicamente los tiempos activos de trabajo, es decir, aquellos en los que se desarrolla una actividad concreta y que van más allá de la mera presencia en el hogar.

1. A este respecto, el Real Decreto que regula el Régimen Especial de Empleo de Hogar indica lo siguiente: «el/la empleador/a y el/la empleado/a podrá acordar tiempos de presencia de éste/a en situación de disponibilidad sin realizar un trabajo concreto, así como la correspondiente retribución [...]» (RD 1424/85) 
Tabla 1. Lista de actividades precodificadas realizadas durante el trabajo de cuidado. EUTPC, 2008

\begin{tabular}{|c|l|}
\hline \multicolumn{2}{|c|}{ Cuidados socio-sanitarios } \\
\hline 1 & $\begin{array}{l}\text { Ayudar en la preparación y provisión de medicamentos (pastillas, supositorios, } \\
\text { etc.) }\end{array}$ \\
\hline 2 & Curas, cambio de vendajes, limpiar heridas, administrar enemas, etc. \\
\hline 3 & Cambiar pañales \\
\hline 4 & Ayudar a hacer ejercicios mentales o físicos \\
\hline \multicolumn{2}{|c|}{ Ayudas personales en el hogar } \\
\hline 5 & Ayudar a comer/beber \\
\hline 6 & Ayudar a vestirse/desvestirse \\
\hline 7 & $\begin{array}{l}\text { Ayudar a asearse/arreglarse (lavar cara y manos, peinar, afeitar, maquillar, cortar } \\
\text { uñas de pies y manos, etc.) }\end{array}$ \\
\hline 8 & Ayudar a andar por la casa o moverse \\
\hline 9 & Ayudar a acostarse/levantarse de la cama \\
\hline 10 & $\begin{array}{l}\text { Ayudar a utilizar el retrete (llevar y sentar en el váter, quitar y poner la ropa, } \\
\text { limpiar tras la deposición, evitar que se manche) }\end{array}$ \\
\hline 11 & Ayudar a ducharse o bañarse (todo el cuerpo) \\
\hline \multicolumn{2}{|c|}{ Actividades domésticas } \\
\hline 12 & Hacer compras \\
\hline 13 & Preparar comidas \\
\hline 14 & Limpiar la casa/cocina \\
\hline 15 & Cuidados a niños \\
\hline 16 & Arreglos/mascotas/jardín \\
\hline 17 & Administrar el dinero/gestiones \\
\hline & \multicolumn{2}{c}{ Acompañamiento dentro y fuera del hogar } \\
\hline 18 & Estar con él/ella en casa sin hacer nada/viendo la televisión \\
\hline 19 & $\begin{array}{l}\text { Estar con él/ella en casa jugando a las cartas, juegos de mesa, leyéndole periódicos, } \\
\text { revistas o libros, etc. }\end{array}$ \\
\hline 20 & Dar un paseo con él/ella \\
\hline 21 & Utilizar el transporte público con él/ella \\
\hline 22 & $\begin{array}{l}\text { Acompañar en actividades de ocio fuera de casa (ir a la cafetería, cine, teatro, de } \\
\text { compras, etc.) }\end{array}$ \\
\hline
\end{tabular}

\section{Resultados}

\subsection{El tiempo de cuidado}

Investigaciones precedentes han diferenciado a las cuidadoras de personas dependientes según su régimen de trabajo: internas, externas o por horas (IMSERSO, 2005a). A estos perfiles, esta investigación añade una figura 
complementaria: la de quienes atienden en régimen de interna exclusivamente durante los fines de semana. El régimen de trabajo determina la cantidad de tiempo que estas trabajadoras invierten en cuidado y condiciona intensamente el resto de actividades de su vida cotidiana (tiempo libre, transporte, tiempo dedicado a otros trabajos, etc.).

Las cuidadoras internas viven (duermen y reciben manutención) en el domicilio de la persona a la que cuidan y le proveen atención en una franja horaria indeterminada. A pesar de que la normativa básica estipula un descanso para las internas de dos horas para las comidas y de ocho horas de sueño entre una jornada y otra, el hecho de residir en la misma vivienda donde trabajan hace que, en muchas ocasiones, esta frontera temporal para el descanso no exista. En muchos casos, no es su jornada de trabajo la que se ajusta a su tiempo de descanso, sino a la inversa:

«Como interna... ¿sabe lo que pasó? La familia era muy buena, pero se necesitaba mucho cariño, mucho amor, mucho cuidado, mucha responsabilidad... Yo trabajaba allí todo el día, estaba pendiente de la señora 24 horas.» Rumana, 46 años.

Existe un grupo de cuidadoras internas que atienden a personas mayores durante los fines de semana y/o días festivos. Su tiempo de trabajo coincide con los días libres de los cuidadores principales, ya sean internos, externos o cuidadores familiares, con el fin de cubrir su tiempo de descanso. El trabajo de las internas de fin de semana se desarrolla en jornadas de 12 a 24 horas de atención continuada y cumple una función de bisagra para la consecución del tiempo libre del resto de cuidadores.

Las cuidadoras externas se caracterizan por no residir en su lugar de trabajo. Son cuidadoras externas a tiempo completo quienes realizan jornadas entre 7 y 12 horas, frecuentemente repartidas entre la mañana y la tarde. Cuando los horarios se prolongan en exceso es posible hablar de falsas externas (Martínez Veiga, 2000: 91): trabajadoras con un régimen de externa, puesto que viven fuera de la casa del empleador, pero que experimentan el resto de condiciones de un trabajo interno. Si a una jornada media de más de 12 horas se suma el tiempo de desplazamiento, el resultado es que estas trabajadoras están en su domicilio unas pocas horas para descansar entre una jornada laboral y la siguiente:

«Me dijeron "este es el horario de trabajo y lo que tienes que hacer", y el problema es que vivo muy lejos y necesitaría salir a las 6 de la mañana de mi casa para llegar a las 8 allí, luego termino sobre las 5 o 6 , pero ahora quiere que trabaje de 8 a 8 . Pero si salgo a las 8 , llego a las 10 a mi casa y ya no tengo espacio para nada...» (Mujer boliviana, 44 años) 
Tabla 2. Tiempo de cuidado según tipo de estimación y régimen de cuidado (horas y centésimas de hora)

\begin{tabular}{|l|c|c|c|c|c|}
\hline & \multicolumn{2}{|c|}{ Diario } & \multicolumn{2}{c|}{ Encuesta de actividades } & \multirow{2}{*}{ N } \\
\cline { 2 - 5 } & Al día $^{1}$ & Semanal & Al día & Semanal & \\
\hline Externas por horas & 4,09 & 20,45 & 4,39 & 21,95 & 8 \\
\hline $\begin{array}{l}\text { Externas a jornada } \\
\text { completa }\end{array}$ & 10,53 & 63,18 & 8,39 & 50,34 & 3 \\
\hline Internas de fin de semana & 22,25 & 44,50 & 9,25 & 18,50 & 3 \\
\hline Internas & 21,90 & 131,40 & 10,23 & 61,38 & 10 \\
\hline Total & 14,59 & 75,03 & 7,93 & 41,50 & 24 \\
\hline
\end{tabular}

1. La estimación corresponde al tiempo semanal de cuidado y supervisión dividido por 5 en «externa por horas», por 6 en «externas a jornada completa»e «internas» y por 2 en "internas de fin de semana".

2. La estimación corresponde al último día que trabajó la entrevistada.

Fuente: Encuesta piloto sobre «Uso del tiempo de los cuidadores de origen extranjero en la Comunidad de Madrid 2008»

Son externas por horas aquellas cuidadoras que trabajan entre una y 6 horas diarias en un mismo hogar. La labor de estas trabajadoras se asemeja a la que desarrollada por el Servicio Público de Atención a Domicilio (SAD)2. Frente a los límites horarios de los recursos formales del SAD, las trabajadoras por horas cuidan en aquellos espacios de tiempo que no pueden ser cubiertos por la familia o por los recursos formales. Este servicio es asequible para hogares que no pueden pagar a una interna o a una externa fija, y su uso se ha incrementado en toda Europa (IMSERSO, 2005c). La flexibilidad de horarios y actividades es un rasgo común de estas cuidadoras:

«Trabajo por horas. Familias de personas mayores... Trabajo en dos casas con personas mayores. Los lunes, miércoles y viernes, entre las 9 y las 12 en una casa, y los martes y jueves, en otra casa.» (Mujer rumana, 46 años)

A través del tiempo invertido en cuidado y en las diferentes actividades que lo componen, es posible inferir su cantidad e intensidad y aproximarse a sus efectos en los cuidadores, las personas cuidadas y su entorno social. De acuerdo con el diario, el tiempo medio de cuidado asciende a 14,6 horas diarias

2. El SAD se define como «un programa individualizado, de carácter preventivo y rehabilitador, en el que se articulan un conjunto de servicios y técnicas de intervención profesional consistentes en atención personal, doméstica, de apoyo psicosocial y familiar y relaciones con el entorno, prestados en el domicilio de una persona en situación de dependencia con el objetivo básico de favorecer la autonomía personal en su medio habitual de vida». (Deloitte, 2008: 9) 
(Tabla 2). La dedicación difiere sustancialmente según elrégimen de trabajo. Las externas por horas dedican un promedio de 4,1 horas diarias, las externas a jornada completa, 10,5 horas; y las internas, en torno a las 22 horas. Según la encuesta de actividades, que no contabiliza los tiempos de presencia, las externas por horas dedican un promedio de 4,4 horas diarias, las externas a jornada completa, 8,4 horas; las internas de fin de semana, 9,3 horas al día; y las internas, un promedio de 10,2 horas.

El cuidado, como actividad general, se descompone en tareas concretas, que se desarrollan sobre todo en función a las necesidades de la persona que demanda la atención. Se trata de actividades frecuentemente imprevisibles que resultan difíciles de planificar, tal y como refleja el discurso de las cuidadoras:

«Sí, entre las seis menos cuarto y seis y media... pero esto depende de su estado de nervios, tengo que darle agua y llevarle al servicio. A las siete y media, de nuevo, tengo que darle el yogur, el agua... Con lo cual, a las diez y media le doy de nuevo la leche y la magdalena, le baño... todo, todo, todo, le acuesto, decimos el Padre Nuestro, hasta que se duerme. Si se levanta por la noche, me despierto, porque hay noches cuando no duerme. Lleva como tres semanas sin dormir...se hace caca encima, le tengo que lavar de noche...» (Mujer rumana, 51 años)

Las actividades de cuidado pueden ser agrupadas en cuatro grandes categorías: servicio doméstico y gestión del hogar, acompañamiento (dentro y fuera del hogar), ayudas relativas al cuidado personal y cuidados socio-sanitarios (Tabla 1). El tiempo dedicado a cada una de estas categorías varía según el régimen de trabajo de las cuidadoras y el nivel de dependencia de la persona mayor. Se puede establecer una tipología de cuidadoras en función de las tareas concretas en que se traduce su tiempo de trabajo. Con este objetivo, se ha realizado un análisis de conglomerados (o análisis cluster) en base al tiempo dedicado a diferentes actividades, del que han resultado dos tipos de cuidadoras $^{3}$. La Tabla 3 muestra el tiempo promedio dedicado a diferentes actividades según tipo.

El tipo 1, «acompañante-ama de casa», realiza fundamentalmente AIVD, es decir, actividades domésticas (hacer la compra, preparar comidas y limpiar) y de acompañamiento. Se caracteriza por cuidar a una persona con un nivel de autonomía relativamente alto y con menores necesidades de apoyo directo, por lo que no suelen proveer cuidados socio-sanitarios ni ayudas personales. Dos de cada tres cuidadoras responden a este perfil.

3. El análisis de conglomerados de k medias estableció dos tipos, aunque una muestra más amplia podría dar lugar a un número mayor. 
Tabla 3. Promedio de horas y centésimas de hora dedicadas a diferentes actividades según tipo de cuidadora

\begin{tabular}{|l|c|c|c|}
\hline & $\begin{array}{c}\text { Acompañante- } \\
\text { ama de casa } \\
(\mathrm{N}=16)\end{array}$ & $\begin{array}{c}\text { Enfermera- } \\
\text { ama de casa } \\
(\mathrm{N}=8)\end{array}$ & Sig. \\
\hline $\begin{array}{l}\text { Ayudar en la preparación y provisión } \\
\text { de medicamentos }\end{array}$ & 0,06 & 0,25 & $* * 0,033$ \\
\hline $\begin{array}{l}\text { Curas, cambio de vendajes, limpiar } \\
\text { heridas, administrar enemas, etc. }\end{array}$ & 0,00 & 0,03 & 0,162 \\
\hline Cambiar pañales & 0,00 & 0,78 & $* * * 0,003$ \\
\hline $\begin{array}{l}\text { Ayudar a hacer ejercicios mentales o } \\
\text { físicos }\end{array}$ & 0,13 & 0,35 & 0,122 \\
\hline Ayudar a comer, beber & 0,00 & 0,99 & $* * * 0,002$ \\
\hline Ayudar a vestirse, desvestirse & 0,12 & 0,65 & $* * * 0,001$ \\
\hline Ayudar a asearse, arreglarse & 0,06 & 0,35 & $* * * 0,002$ \\
\hline Ayudar a andar por la casa o moverse & 0,04 & 0,19 & $* * 0,018$ \\
\hline $\begin{array}{l}\text { Ayudar a levantarse, acostarse en la } \\
\text { cama }\end{array}$ & 0,02 & 0,17 & $* * * 0,001$ \\
\hline Ayudar a utilizar el retrete & 0,03 & 0,48 & $* * * 0,000$ \\
\hline Ayudar a ducharse o bañarse & 0,16 & 0,21 & 0,681 \\
\hline Hacer compras & 0,63 & 0,13 & $* * 0,020$ \\
\hline Preparar comidas & 1,16 & 1,18 & 0,972 \\
\hline Limpiar la casa, cocina & 2,20 & 1,04 & 0,072 \\
\hline Cuidados a niños & 0,00 & 0,01 & 0,162 \\
\hline Arreglos, mascotas, jardín & 0,26 & 0,33 & 0,774 \\
\hline Administrar el dinero, gestiones & 0,00 & 0,13 & 0,162 \\
\hline $\begin{array}{l}\text { Estar con él/ella en casa sin hacer nada } \\
\text { o viendo la tele }\end{array}$ & 1,12 & 0,95 & 0,746 \\
\hline $\begin{array}{l}\text { Estar con él/ella en casa jugando a las } \\
\text { cartas, juegos de mesa, leyéndole, etc. }\end{array}$ & 0,53 & 0,14 & 0,280 \\
\hline Dar un paseo con él/ella & 0,60 & 1,06 & 0,135 \\
\hline $\begin{array}{l}\text { Utilizar el transporte público con él o } \\
\text { ella }\end{array}$ & 0,05 & 0,00 & 0,345 \\
\hline $\begin{array}{l}\text { Acompañar en actividades de ocio } \\
\text { fuera de casa }\end{array}$ & 0,00 & 0,06 & 0,162 \\
\hline & & & \\
\hline
\end{tabular}

$* * \mathrm{p}<0,05 * * * \mathrm{p}<0,01$.

Fuente: Encuesta piloto sobre «Uso del tiempo de los cuidadores de origen extranjero en

la Comunidad de Madrid 2008» 
El tipo 2, «enfermera-ama de casa», se caracteriza por realizar un amplio abanico de actividades, cuyo núcleo diferenciador lo forman los cuidados socio-sanitarios (provisión de medicamentos, cambiar pañales, etc.) y las ayudas personales en el hogar (ayudar a comer/beber, desplazarse, vestirse, etc.). También suelen realizar actividades domésticas y dedicar tiempo a acompañamiento. Estas cuidadoras atienden a personas con peor estado de salud y mayor nivel de dependencia. Generalmente, este tipo de atención coincide con la etapa previa o inmediatamente posterior al ingreso en un hospital o residencia. Una de cada tres cuidadoras se encuadra en el este tipo.

\subsection{La articulación entre trabajo y vida privada}

Los resultados que se presentan a continuación analizan, con información procedente del diario, el modo en que el trabajo de cuidado está presente en la vida cotidiana de estas mujeres. En concreto, responden a dos cuestiones: qué proporción de tiempo ocupa el cuidado en relación con su tiempo total disponible y cómo se distribuye el cuidado a lo largo de la semana. Tanto la distribución del tiempo de cuidado semanal como su presencia con respecto al resto de actividades muestran las diferentes posibilidades de articulación entre la vida laboral y personal de las cuidadoras. El principal elemento de diferenciación entre estas trabajadoras es el régimen laboral, que tiene un intenso impacto en la distribución del tiempo semanal en actividades (figura 1).

Figura 1. Distribución del tiempo semanal en actividades de las cuidadoras, según el régimen de cuidado (\%)

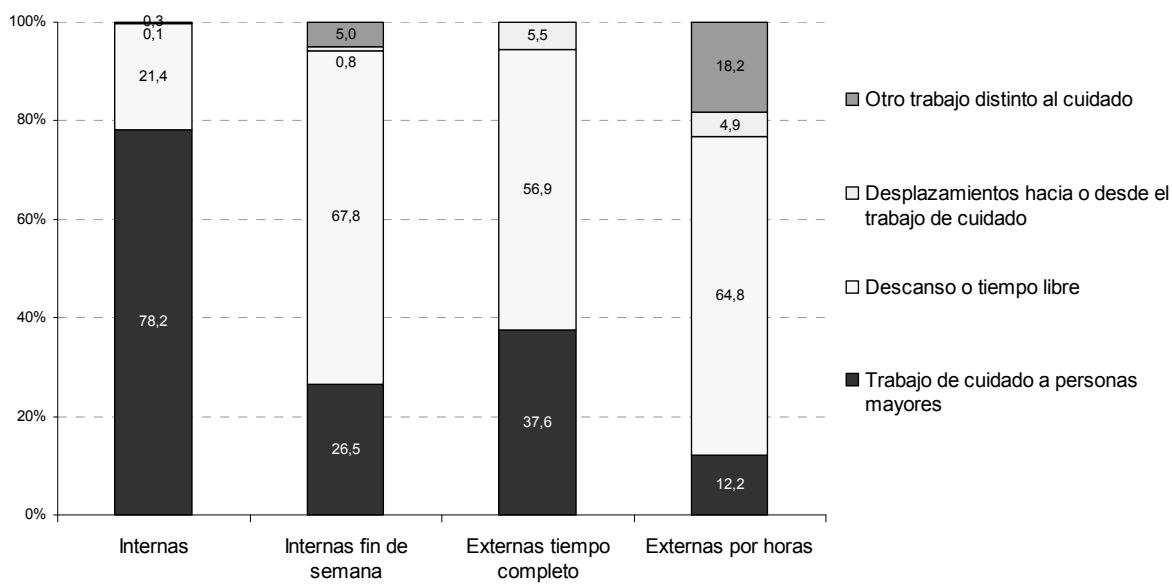

Fuente: Encuesta piloto sobre «Uso del tiempo de los cuidadores de origen extranjero en la Comunidad de Madrid 2008» 
Figura 2. La distribución de las actividades según el momento de la semana Cuidadoras internas

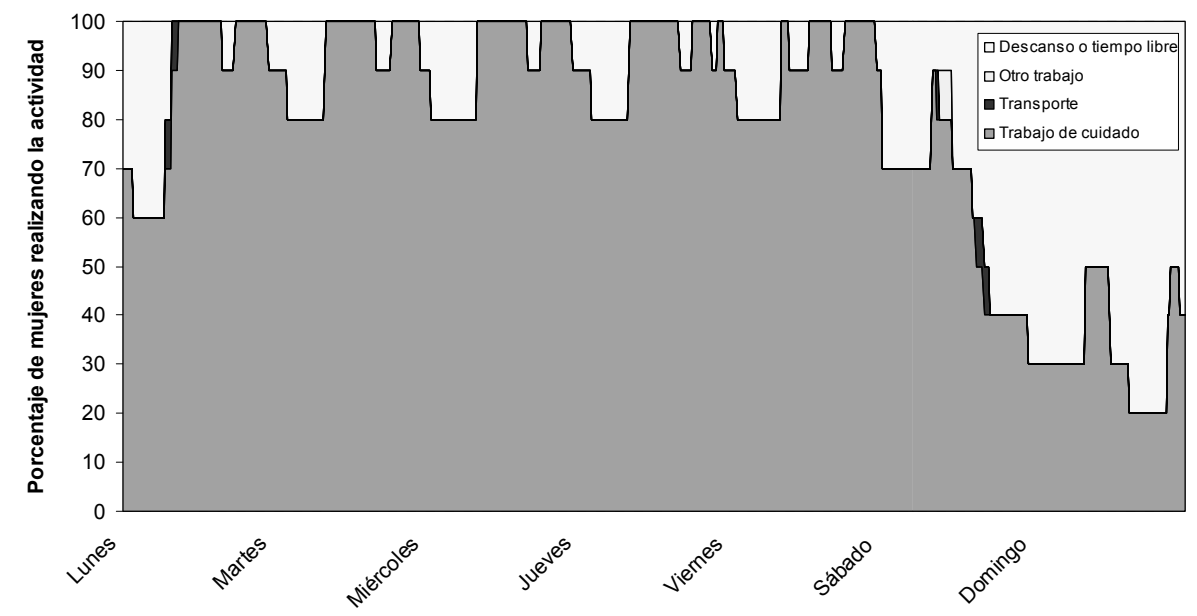

Fuente: Encuesta piloto sobre «Uso del tiempo de los cuidadores de origen extranjero en la Comunidad de Madrid 2008»

El grupo con rasgos más diferenciados es el de las cuidadoras internas. En este caso, el tiempo cotidiano está intensamente desequilibrado hacia el trabajo remunerado, con jornadas laborales muy largas y una dedicación a las necesidades propias (descanso, ocio y relaciones sociales) muy reducida. Este grupo dedica 131,4 horas semanales al trabajo (el $78 \%$ de su tiempo) y 35,9 horas a descanso y tiempo libre (el $21 \%$ ) (Figura 1). El poco tiempo libre que tienen se concentra en los fines de semana y, como se observa en la Figura 2, en torno a la mitad de las cuidadoras pasan las noches del sábado al domingo y del domingo al lunes fuera del hogar de cuidado.

Las propias cuidadoras internas consideran la escasez de tiempo libre como uno de sus principales problemas, que relacionan con la falta de independencia y autonomía. Son conscientes de que sus largas jornadas limitan las posibilidades de realización de otras actividades y, por tanto, de establecer y mantener relaciones sociales estrechas fuera del ámbito de su empleo. Perciben que buena parte de su tiempo no les pertenece porque les ha sido arrebatado:

«No, para mí, sobre todo, sería la falta de, podría decir, independencia, porque uno no es dueño de su tiempo, ya, claro, si tú trabajas no eres en este momento dueño de tu tiempo, pero después de que tú termines tu trabajo ya tú eres dueño de tu tiempo, ya tu tiempo tú lo planificas, puedes decidir estudiar, o puedes decidir salir, o puedes ir donde una amiga, o sea, puedes decidir, pero si trabajas de interna no es así.» (Mujer dominicana, 47 años) 
Las trabajadoras externas de jornada completa reflejan un uso del tiempo con un mayor equilibrio entre las distintas actividades, en el que el tiempo libre supera al tiempo de trabajo: dedican 63,2 horas al trabajo (un $38 \%$ de su tiempo semanal), a lo que hay que agregar 9,3 horas de transporte hacia o desde el hogar de trabajo (un $6 \%$ del tiempo) (Figura 1). Disponen de 95,6 horas de tiempo libre (un $55 \%$ ). A pesar de ello, su jornada laboral es especialmente extensa si se compara con las 40 horas (el $24 \%$ del tiempo semanal) que establece la normativa básica del personal al servicio del hogar familiar. Este colectivo cuenta, sin embargo, con situaciones heterogéneas que se corresponden con lo que se ha calificado de falsas externas, entre quienes la necesidad de cubrir sus gastos de manutención y los bajos salarios característicos del sector les llevan a prolongar su jornada laboral por encima de las 10 horas diarias.

La Figura 3 muestra la distribución del tiempo de las externas a jornada completa a lo largo de la semana. Sus horas libres coinciden con el final de la tarde y la noche, a excepción de los domingos por la tarde, cuando todas las trabajadoras entrevistadas reportaron disponer de tiempo libre. Esta distribución del tiempo es muy similar a la de la mayoría de trabajadores de otros sectores (INE, 2007).

Muchas externas a jornada completa se enfrentan a una difícil articulación entre su vida laboral y personal, pues al no residir en el domicilio de la

Figura 3. La distribución de las actividades según el momento de la semana Cuidadoras externas a jornada completa de 7 a 12 horas diarias

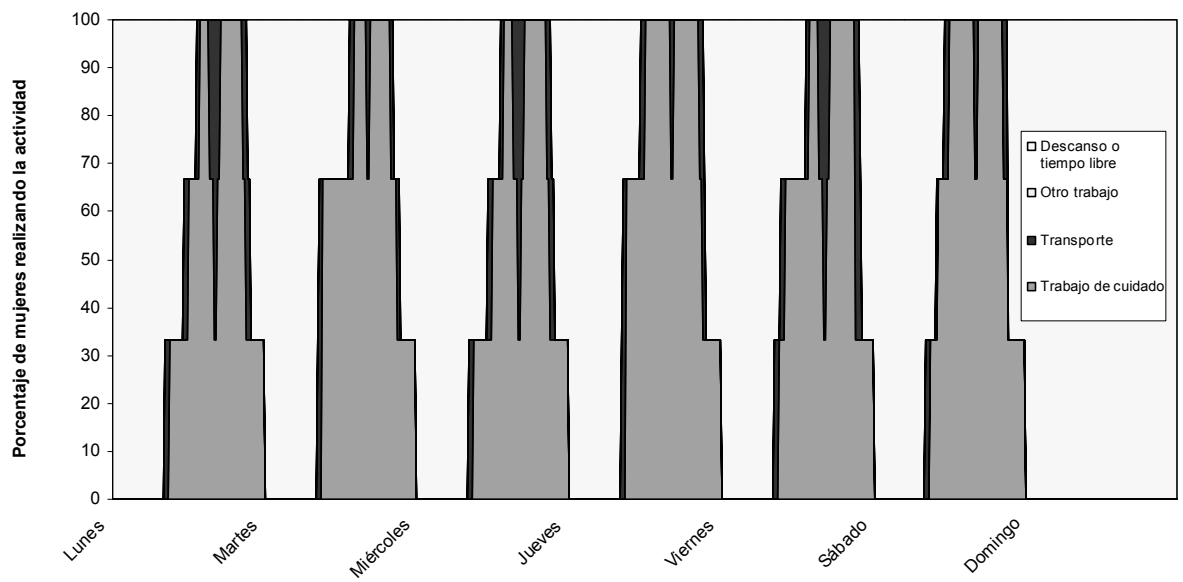

Fuente: Encuesta piloto sobre «Uso del tiempo de los cuidadores de origen extranjero en la Comunidad de Madrid 2008» 
persona mayor han de afrontar la doble atención que supone su actividad laboral y sus obligaciones domésticas. Su carga global de trabajo (García Sainz, 1998) -agregado de trabajo remunerado y no remunerado- es muy elevada y, en muchas ocasiones, genera estrés y una percepción del tiempo como recurso escaso, tal y como se refleja en el siguiente fragmento de entrevista:

«Estoy deseando descansar porque siempre me falta algo, si no es barrer, es recoger y si no es una cosa es la otra, pero siempre ando con algo que me falta, ya voy poniendo lavadoras que digo bueno luego tengo que acabar de hacer la comida, luego tengo que dar una vuelta ir a dejar el ordenador, luego tengo que ir al trabajo, antes de eso tengo que ir donde la Seguridad Social a dejar un papel, bueno, tengo todas las horas ocupadas a veces no llego a una o a la otra.» (Mujer ecuatoriana, 31 años)

Las externas por horas son el grupo que cuenta con la jornada de cuidado más reducida, aunque la complementan con otro tipo de trabajo (Figura 1). Dedican al cuidado 20,4 horas (el $12 \%$ ) y 30,6 horas a otros trabajos que no son cuidado (el $18 \%$ ). Buena parte de su tiempo libre o de descanso es reinvertido en otras actividades laborales. Según la EPUTC 2008, la mitad de las externas por horas realiza otro trabajo. Al igual que extender la jornada laboral es, para las externas con jornada completa, una estrategia para aumentar sus ingresos, las externas por horas recurren al pluriempleo como mecanismo para reunir el salario necesario. El tiempo libre constituye el $65 \%$, con 108,8 horas a la semana, y dedican a transporte 8,2 horas semanales, el $5 \%$ de su tiempo semanal.

El trabajo de cuidado de las externas por horas se distribuye fundamentalmente en las horas de la mañana (Figura 4). Los trabajos complementarios al cuidado se desarrollan también durante esos periodos, aunque en algunos casos ocupan noches y fines de semana. El resultado es que la totalidad de estas mujeres trabaja en algún momento de las mañanas de los días laborables, ya sea cuidando o realizando otras actividades. Durante las tardes y los fines de semana disponen de más tiempo libre. Los relatos de estas trabajadoras reflejan las principales motivaciones para afrontar este tipo de trabajo. En primer lugar, plantean como razón la necesidad económica:

«Estoy cuidando dos personas. Una, durante el día y otra aquí, en el hospital durante la noche. Lo que pasa es que el sueldo no es... lo que dicen según las bolsas o el mínimo que se debería pagarnos es 600 euros. Pero si calculamos que una habitación son 300 euros, el transporte el abono de metro son 50 o 55, más 150 para comer mínimamente, y luego están las cosas personales, detergentes, champús... no te alcanza con los 600 euros.» (Mujer boliviana, 44 años)

En segundo lugar, conciben este tipo de trabajo como un medio para conciliar vida familiar y laboral: 
«Ahora trabajo por horas. Tengo varios trabajos, porque al venir mi hijo, necesito cuidar de él un poco, aunque él se hace solo la comida y sabe poner la lavadora...» (Mujer rumana, 38 años)

Y en tercer lugar, algunas cuidadoras se plantean el objetivo de disfrutar de su tiempo libre reinvirtiéndolo en otro tipo de actividades formativas o de ocio:

«Mira, ahorita el trabajo que me gusta con la empresa, es bueno porque es por horas, entonces claro yo trabajo las noches, que las pagan muy bien, trabajo como cuidadora de personas mayores tanto en casas como convalecientes, enfermos, y ese trabajo lo pagan muy bien, o no lo pagan bien, o es sobre todo por la disponibilidad de tiempo, como a mí me gusta hacer cosas, entonces claro, las noches para mí, aunque trabaje diez horas no me importa porque tengo disponibilidad para hacer cosas de día, hacer cursos, he hecho cursos en la EMSI con la Comunidad de Madrid, hago voluntariado en la Cruz Roja, entonces me gusta hacer muchas cosas.» (Mujer venezolana, 34 años)

Las internas de fin de semana conforman el grupo con una distribución del tiempo más atípica. Disponen de 113,8 horas de tiempo libre o descanso a la semana (el $68 \%$ de su tiempo), frente a las 52,8 horas de trabajo remunerado (el $32 \%$ ). A pesar de dedicar parte de su tiempo libre a otros trabajos, éstos apenas representan el $6 \%$ de su tiempo semanal (Figura 1). Su uso del tiempo sigue una lógica inversa al resto de trabajadores: concentran la práctica totalidad de su trabajo de cuidado durante el fin de semana (44,5 horas), y es en

Figura 4. La distribución de las actividades según momento de la semana

Cuidadoras externas por horas

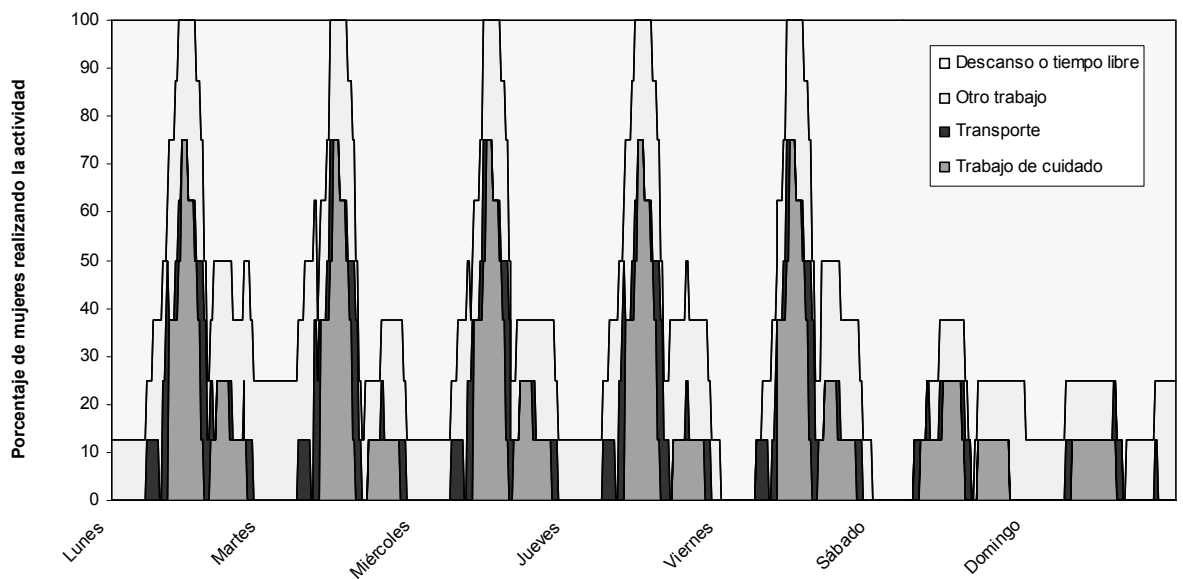

Fuente: Encuesta piloto sobre «Uso del tiempo de los cuidadores de origen extranjero en la Comunidad de Madrid 2008» 
Figura 5. La distribución de las actividades según el momento de la semana

Cuidadoras externas fin de semana (Más de 12 horas diarias en fin de semana)

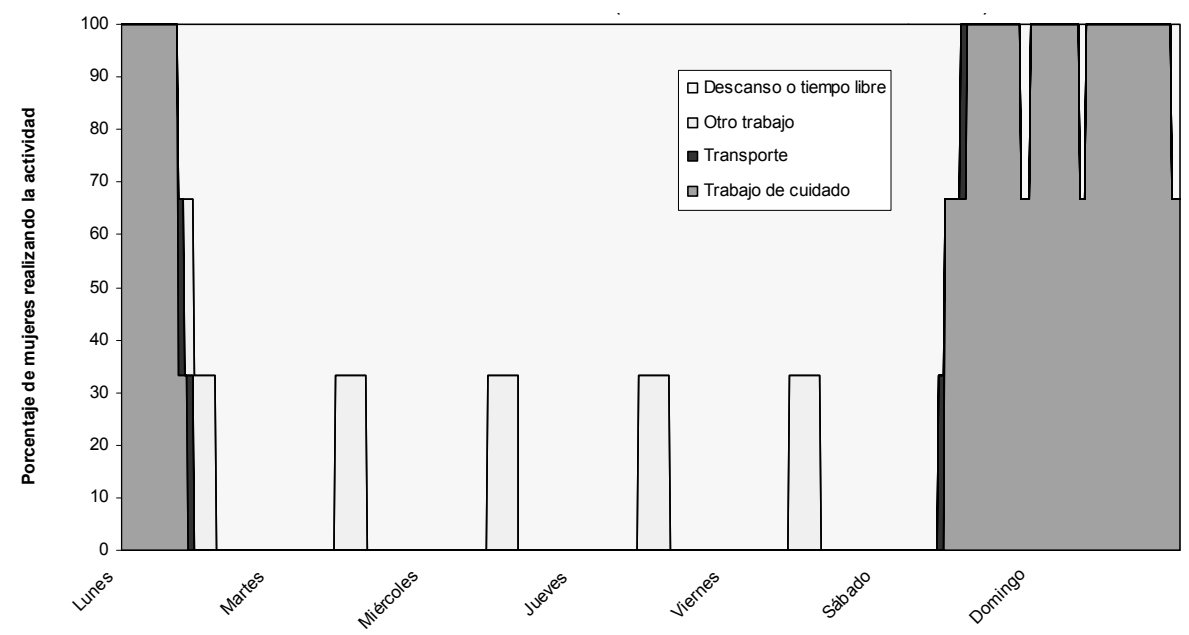

Fuente: Encuesta piloto sobre «Uso del tiempo de los cuidadores de origen extranjero en la Comunidad de Madrid 2008»

los días de diario cuando disponen de abundante tiempo libre o de descanso (Figura 5). Esto les confiere una importante distinción, no sólo respecto al resto de trabajadoras del sector, sino también respecto al ritmo del grueso de los trabajadores.

Generalmente, las internas de fin de semana conciben el cuidado como un trabajo esporádico mientras encuentran algo que ofrezca mayores ingresos, o bien son estudiantes que necesitan dinero extra y que no tienen el objetivo de incrementar su tiempo de trabajo. Estas trabajadoras suelen reemplazar a las cuidadoras fijas durante su tiempo libre. Se podría decir, utilizando la expresión empleada por los servicios formales, que es una figura que facilita el respiro familiar de los cuidadores principales, ofreciéndoles un apoyo indirecto para que puedan hacer compatible su vida laboral y su vida personal.

\section{Conclusiones}

Este trabajo aporta nueva información sobre las condiciones de vida y de trabajo de las cuidadoras inmigrantes de personas mayores en los hogares, un colectivo con una importancia social y económica creciente en España. En concreto, se han ofrecido datos sobre el uso del tiempo de estas cuidadoras en la Comunidad de Madrid, desde una perspectiva cuantitativa (tiempo 
dedicado a cuidado y a otras actividades y ciclo semanal del trabajo de cuidado) y cualitativa (discursos de las cuidadoras sobre su tiempo de trabajo). Los resultados obtenidos profundizan sobre las diferencias internas de este colectivo en relación con su régimen laboral.

Con respecto al tiempo de trabajo de las cuidadoras, los resultados de la EPUTC 2008 son similares a los hallados por el Colectivo IOÉ para las trabajadoras inmigrantes internas del sector doméstico (Colectivo IOÉ, 2003). Según su estudio, estas mujeres dedicaban en el año 2000 un promedio semanal de 60 horas al trabajo, muy cerca de las 61,4 horas calculadas a partir de la EPUTC 2008 cuando no se contabilizan los tiempos de presencia. Sin embargo, dado que la Organización Internacional del Trabajo establece que las horas efectivamente trabajadas deben incluir «el tiempo transcurrido en el lugar de trabajo esperando, o inactivo por razones tales como la falta ocasional de trabajo [...]» (OIT, 2008: 7), las horas de presencia deben ser computadas. La metodología adecuada para ello es el diario, que con la inclusión de los tiempos de presencia estima que el tiempo semanal de trabajo de las internas asciende a 131,4 horas.

Independientemente de la metodología utilizada, tanto las internas como buena parte de las externas superan con amplitud la jornada semanal ordinaria de 40 horas. En el caso de las externas, el análisis de la jornada semanal de trabajo ha revelado que, en muchos casos, no se cumple la normativa vigente que estipula un máximo de 9 horas diarias de trabajo efectivo y un descanso de por lo menos 10 horas entre una jornada laboral y otra (BOE 13/08/1985). A la generalizada vulneración de la legislación laboral española se añade la percepción negativa de las propias cuidadoras sobre sus condiciones laborales. El análisis de su discurso pone de manifiesto que aquellas que tienen largas jornadas se encuentran insatisfechas con su uso del tiempo, lo que coincide con investigaciones precedentes que indican que la falta de tiempo libre de las cuidadoras incrementa su estrés (Gallart, 2007). Es conveniente, por tanto, articular mecanismos que garanticen a este colectivo los derechos laborales del resto de trabajadores y contribuyan a mejorar su calidad de vida ${ }^{4}$.

En cuanto al tipo de actividades que se desarrollan, la presencia mayoritaria de trabajadoras que apenas proveen cuidados socio-sanitarios y/o ayudas

\footnotetext{
4. Naciones Unidas INSTRAW promovió a finales del 2009 la apertura de un proceso de diálogo entre las asociaciones de mujeres trabajadoras del hogar, el movimiento feminista, el Gobierno y los sindicatos, con el fin de avanzar en la toma de decisiones respecto a la situación de las empleadas de hogar migrantes. Las conclusiones de los encuentros y otros documentos de interés pueden descargarse en: http://www.un-instraw.org/es/md/ md-homepage/migration-and-development.html.
} 
en el hogar (categorizadas como acompañantes-amas de casa) expresa que las familias demandan más ayuda para realizar las tareas domésticas y que la mayoría de las cuidadoras se ocupa de personas con un cierto nivel de autonomía, aunque con necesidades puntuales y que requieren supervisión (Rogero García y Martín Coppola, 2010). La importante presencia de tareas que no son propiamente cuidados socio-sanitarios lleva a cuestionarse dónde reside la especificidad del trabajo de cuidado.

A pesar de que, en la práctica, los cuidados directos y las actividades domésticas suelen alternarse e incluso superponerse, ambos tipos de trabajo son sustantivamente diferentes: tienen distinta consideración social, implican un esfuerzo y formación diferentes y su valoración en el mercado no es la misma. La definición del trabajo de cuidado, de este modo, debe residir no tanto en las actividades en sí como en para quién se realizan. Debe hablarse de cuidado sólo en aquellos casos en que las tareas se realizan para personas que no pueden desarrollarlas por ellas mismas. La función de las cuidadoras es, además de proporcionar ayudas físicas directas, estar disponibles para proveer apoyos puntuales cuando sea necesario. Como se ha indicado, muchas de las ayudas personales en el hogar y de los cuidados socio sanitarios tienen un carácter imprevisible, que requiere de una supervisión constante, como por ejemplo cambiar pañales o acompañar al baño.

Los resultados de este trabajo confirman también las grandes diferencias en las condiciones de trabajo de las cuidadoras según su régimen laboral. Las internas son, debido a su extensa dedicación a cuidado y a sus grandes limitaciones temporales para desarrollar otras actividades, las trabajadoras más vulnerables del sector. Mientras las internas circunscriben su horizonte de preocupaciones al ámbito de su actividad laboral, como reflejo de su posición dentro de un sector que tiende a aislarlas del conjunto de la vida social, entre las externas los discursos hacen referencia a un mayor desempeño de papeles en la sociedad (en tanto que mujeres, trabajadoras, inmigrantes, esposas, madres, etc.), lo que remite a una mayor libertad para desarrollarse como ciudadanas y para integrarse en los modos de vida generalizados.

Las principales limitaciones de este trabajo residen en el número de casos de la encuesta. La EPUTC 2008 tiene un carácter exploratorio y la capacidad de su muestra para representar estadísticamente al colectivo inmigrante que cuida mayores en la Comunidad de Madrid es limitada. No obstante, esta fuente tiene una representatividad intencional basada en estudios precedentes y un elevado nivel de detalle y se complementa con una extensa muestra de entrevistas cualitativas. Estas características y la escasez de información cuantitativa sobre este colectivo concede a los resultados de este trabajo un valor 
especial. De cara a futuros estudios, se recomienda la utilización de muestras más amplias. Sería positivo, en este aspecto, crear un registro público de estas trabajadoras que permitiera cuantificar de manera básica este fenómeno, ofreciendo a las Administraciones Públicas información sobre su presencia en los hogares españoles.

\section{Bibliografía}

BITTMAn, M., J. FAST, K. FisHER, y C. THOMSON, «Making the invisible visible: the life and time(s) of informal caregivers», en Family Time: The Social Organisation of Care, N. Folbre y M. Bittman (Eds.), Londres y Nueva York, Routledge, 2004.

Boletín Oficial del Estado (13/08/1985). Real Decreto 1424/1985, de 1 de agosto, por el que se regula la relación laboral de carácter especial del servicio del hogar familiar.

Colectivo IOÉ, «Situación laboral de las mujeres inmigrantes no comunitarias», en Los inmigrantes y el mundo del trabajo, Tornos Cubillos, Madrid, Universidad Pontificia de Madrid, 2003.

Deloitte, Perspectivas de futuro de los servicios de atención a la dependencia. El Servicio de Atención a Domicilio (SAD). Estudio del marco conceptual, oferta, demanda y modelo de costes, 2008.

Eurostat, Harmonised European Time Use Surveys, Luxemburgo, Eurostat Methodologies and Working Papers. Eurostat, 2008.

Gallart Fernández-PUebla, A., Sobrecarga del cuidador inmigrante no profesional formal en Barcelona, tesis doctoral, Facultad de Humanidades, Universitat Internacional de Catalunya, San Cugat, 2007.

GARCía SAINZ, C., La carga global de trabajo. Un análisis sociológico, tesis doctoral, Departamento de Cambio Social (Sociología I), Universidad Complutense de Madrid, Madrid, 1998.

IMSERSO, Cuidado a la dependencia e inmigración, Madrid, Ministerio de Trabajo y Asuntos Sociales, 2005a.

- Cuidados a las personas mayores en los hogares españoles. El entorno familiar, Madrid, Instituto de Mayores y Servicios Sociales, 2005b.

- Libro Blanco de la Dependencia, Madrid, Ministerio de Trabajo y Asuntos Sociales, 2005c.

- Las Personas Mayores en España, Informe 2006, Madrid, Ministerio de Trabajo y Asuntos Sociales, 2006.

INE, Encuesta de Empleo del Tiempo. Qué hacemos y durante cuánto tiempo, Cifras INE, Boletín Informativo del Instituto Nacional de Estadística, Madrid, INE, 2007. 
-Proyecto metodológico de la Encuesta de Empleo del Tiempo 2002-2003, INE, Madrid, 2003.

MARTínEZ BujÁN, R., «El cuidado de ancianos: un vínculo entre la inmigración y el envejecimiento», en Panorama social, n. ${ }^{\circ}$ 2, 2005, pp. 86-97.

- «La latinoamericanización de la inmigración en España». Cuadernos Geográficos de la Universidad de Granada, n. ${ }^{\circ}$ 36, 2006, pp. 51-64.

MARTíNEZ VeIGA, U., «Evolución y clasificación del trabajo doméstico inmigrante», en OFRIM Suplementos, junio 2000, Madrid.

OIT, «Informe II. Medición del tiempo de trabajo», $18 .{ }^{a}$ Conferencia Internacional de Estadísticos del Trabajo, Ginebra, Organización Internacional del Trabajo, 2008.

ONU, Guía para la elaboración de estadísticas sobre el empleo del tiempo para medir el trabajo remunerado y no remunerado, Nueva York, Departamento de Asuntos Económicos y Sociales, División de Estadística, Naciones Unidas, 2006.

PEDRERO, M., «Propuesta metodológica para medir y valorar el cuidado de la salud doméstico no remunerado», en La economía invisible y las desigualdades de género. La importancia de medir y valorar el trabajo no remunerado, Washington DC, Organización Panamericana de la Salud (OPS), 2008.

Pérez Orozco, A., D. Paiewonsky y M. García Domínguez, Cruzando Fronteras II. Migración y desarrollo desde una perspectiva de género, Madrid, Instituto de la Mujer / UN-INSTRAW, 2008.

Pla Julián, I., Informalidad en el empleo y precariedad laboral de las empleadas de hogar, Valencia, Universidad de Valencia y Ministerio de la Igualdad, 2004.

Rogero GarCía, J. y E. MARTín COPPOLA, «Un estudio exploratorio del cuidado provisto por inmigrantes a mayores de 64 años en los hogares», en Index de Enfermería, (en prensa), 2010.

\section{Nota:}

Este trabajo ha sido realizado en el marco del proyecto de investigación «Inmigración y cuidados de personas mayores en Madrid» (CUIMAD), financiado por la Fundación BBVA, dirigido por Vicente Rodríguez Rodríguez, y cuyo equipo de investigación estuvo formado por Silvia Marcu, Gloria FernándezMayoralas Fernández, Fermina Rojo Pérez, Raúl Lardiés Bosque, Santiago Ramos Espina, Eva Martín Coppola y Jesús Rogero García. 